Published in final edited form as:

Leukemia. 2020 August ; 34(8): 2238-2242. doi:10.1038/s41375-020-0739-7.

\title{
Genomic characterization of diffuse large B-cell lymphoma transformation of nodular lymphocyte-predominant Hodgkin lymphoma
}

\author{
Joo Y. Song ${ }^{1}$, Caoimhe Egan ${ }^{2}$, Alyssa C. Bouska ${ }^{3}$, Weiwei Zhang ${ }^{3}$, Qiang Gong ${ }^{1}$, Girish \\ Venkataraman ${ }^{4}$, Alex F. Herrera ${ }^{5}$, Lu Chen ${ }^{6}$, Rebecca Ottesen ${ }^{7}$, Joyce C. Niland ${ }^{7}$, Victoria \\ Bedell $^{1}$, Maria Valle-Catuna ${ }^{1}$, Joyce Murata-Collins ${ }^{1}$, Dennis D. Weisenburger ${ }^{1}$, Javeed \\ Iqbal $^{3}$, Elaine S. Jaffe ${ }^{2}$, Wing C. Chan ${ }^{1}$ \\ ${ }^{1}$ Department of Pathology, City of Hope National Medical Center, Duarte, CA, USA \\ ${ }^{2}$ Hematopathology Section, Laboratory of Pathology, National Cancer Institute, Bethesda, MD, \\ USA \\ ${ }^{3}$ Department of Pathology, University of Nebraska, Omaha, NE, USA \\ ${ }^{4}$ Department of Pathology, University of Chicago, Chicago, IL, USA \\ ${ }^{5}$ Department of Hematology \& Hematopoietic Cell Transplantation, City of Hope National Medical \\ Center, Duarte, CA, USA \\ ${ }^{6}$ Department of Information Sciences, City of Hope National Medical Center, Duarte, CA, USA \\ ${ }^{7}$ Department of Diabetes and Cancer Discovery Science, City of Hope National Medical Center, \\ Duarte, CA, USA
}

To the Editor:

Nodular lymphocyte-predominant Hodgkin lymphoma (NLPHL) is a rare subtype of lymphoma that usually has an indolent clinical behavior. Similar to classical Hodgkin lymphoma (cHL), the tumor cells in NLPHL represent a minority of the cells in the tumor and, therefore, have been a challenge to study because of insufficient material for analysis. Transformation of NLPHL (tNLPHL) to diffuse large B-cell lymphoma (DLBCL) is seen in up to $30 \%$ of the cases [1] and can have varying morphologic patterns. However, two main patterns are seen, T-cell/histiocyte-rich large B-cell lymphoma (THRLBCL) and more typical DLBCL with sheets of large cells. Because the tumor cells are also sparse in

Joo Y. Song, josong@ coh.org.

Author contributions JYS and WCC designed, performed the research, analyzed the data, and wrote the paper. CE, ACB, WZ, QG, JI, GV, AFH, DDW, and ESJ analyzed the data and edited the paper. LC, JCN, and RO provided the statistical analysis and edited the paper. VB, MV-C, and JM-C performed the research and edited the paper. All authors reviewed the paper. Research reported in this publication included work performed in the Pathology Core and Integrated Genomics Core supported by the National Cancer Institute of the National Institutes of Health under grant number P30CA033572. The content is solely the responsibility of the authors and does not necessarily represent the official views of the National Institutes of Health.

Compliance with ethical standards

Conflict of interest The authors declare that they have no conflict of interest.

Supplementary information The online version of this article (https://doi.org/10.1038/s41375-020-0739-7) contains supplementary material, which is available to authorized users. 
THRLBCL, it has also been difficult to study these cases. However, with laser capture micro-dissection, a limited number of cases of tNLPHL have been investigated by nextgeneration sequencing $[2,3]$ using limited gene panels.

In this study, we evaluated tNLPHL cases that have the sheet-like growth pattern typical of de novo DLBCL. We characterized the genomic landscape of such cases using nextgeneration sequencing and copy number analysis (CNA) to gain a better understanding of the pathogenesis of these tumors.

We identified 19 cases of tNLPHL from three institutions (National Cancer Institute, University of Chicago, and City of Hope National Medical Center). Cases that had a pattern of THRLBCL were excluded to ensure that only cases with typical DLBCL growth pattern were studied. A diagnosis of NLPHL preceded the development of DLBCL in 5 patients (average interval, 4.7 years), whereas both NLPHL and DLBCL were seen concurrently in 11 patients (see Table S1). Three patients developed NLPHL after the diagnosis of DLBCL $(1,7$, and 12 years after). Cases with concurrent NLPHL and DLBCL were macrodissected to obtain NLPHL areas with only a few tumor cells $(<5 \%)(n=8)$. These NLPHL areas were sequenced and used as matched germline DNA for the corresponding DLBCL. This study was approved by the local institutional review board and was conducted in accordance with the Declaration of Helsinki. DNA was extracted from formalin-fixed, paraffin-embedded tissue blocks and targeted sequencing was performed using a 356 gene panel containing the mutations most frequently seen in B-cell lymphoma and Hodgkin lymphoma (see Supplemental Methods and Tables S2, S3, and S4).

There were 14 males and 5 females with tNLPHL included in this study (M:F ratio of 2.8:1). The median age of the patients was 37 years (range, 15-68 years). The majority of cases of DLBCL had nodal involvement (68\%), but other sites included the spleen, submandibular gland, and gastrointestinal tract (Table S1).

We performed CNA on 19 cases of tNLPHL, but one case failed quality control metrics and was excluded. In the remaining 18 cases, we observed that the number of genomic aberrations in these cases was comparable to that seen in de novo DLBCL cases, and almost all chromosomes showed aberrations. Using GISTIC, we found copy number $(\mathrm{CN})$ gains of several oncogenes (REL, BCL11A, BCL6, CARD11, JAK2) and CN loss of the tumor suppressor gene $C D K N 2 A$ (Q-bound $<0.05$ and G-score < 1.0) in tNLPHL (Fig. 1).

Although a number of pathways are identified, we found that genomic abnormalities in tNLPHL were most often associated with the PI3K (SGK1) and NF-kB (CARD11, JUNB) pathways, and chromatin modification regulators (Fig. 2). This mutational spectrum of tNLPHL resembles the DLBCL Cluster 4 of Chapuy et al. [4], which are primarily germinal center B-cell (GCB) type DLBCL. Transformed NLPHL also had frequent mutations in genes not often seen in de novo DLBCL (TET2, JUNB, and NOTCH2) and this provides new insights into the pathogenesis of these rare tumors.

Mutations in the PI3K pathway (SGK1, ZFP36L1, EIF3A, IL7R, and PIK3RI) were common in tNLPHL. Mutations of $S G K 1$ (serum and glucocorticoid-regulated kinase 1; $26 \%$ of cases mutated in our study) have been reported in NLPHL [2] as well as de 
novo DLBCL, particularly those of germinal center origin [5]. The role of $S G K 1$ in B-cell lymphoma is still unclear, but some data suggest that the mutation acts as an oncogene rather than a tumor suppressor gene in lymphoma [2]. Our cases, as well as the two cases seen by Hartmann et al. [2], showed $S G K 1$ mutations particularly between positions 20 and 200 (Fig. S1a). We compared the location and frequency of $S G K 1$ mutations in the three large de novo DLBCL studies [4-6], which found a predisposition for mutations in this region as well. Some studies suggest this region is important for localization of the protein into the mitochondria and nucleus [7]. Mutations and splice mutations in these regions, as seen in our tNLPHL cases, may alter SGK1 localization and change its normal regulatory functions [8].

Mutations in the NF-kB pathway were common in tNLPHL including CARD11, JUNB, BCL10, NFKBIA, and TNFAIP3. JUNB mutations (Fig. S1b) are rarely reported in de novo DLBCL but were frequently detected in our series of tNLPHL (21\%), as well as other studies of NLPHL [2, 3]. JunB is a member of the activator protein-1 (AP-1) family of transcription factors, which collectively regulate genes important for proliferation, apoptosis, differentiation, and the immune response. A study by Szremska et al. [9] using a mouse model showed that JunB inhibits proliferation and transformation of B-cells and, thus, one can hypothesize that inactivation or lack of JunB makes B-cells more susceptible to transformation.

We observed CN gains of REL in 56\% of our cases of tNLPHL, supporting the notion that this gene is important in the pathogenesis of tNLPHL. Heise et al. [10] found that c-RELdeficient germinal center B-cells failed to upregulate genes involved in metabolic functions that allow the cell to meet the energy demands required by rapid proliferation. Genomic gains in $R E L$, as seen in tNLPHL, may contribute to lymphomagenesis by dysregulating cellular metabolism and promoting survival and proliferation [10].

We also observed frequent genetic abnormalities affecting DNA methylation and histone and chromatin modification pathways such as mutations of EP300 (21\% of our cases), CREBBP (16\%) and TET2 (11\%). TET2, is infrequently mutated in DLBCL (6\% of DLBCL, 7\% of GCB DLBCL), but was mutated in $11 \%$ of our cases. Recent work has shown that TET2 should be considered a tumor suppressor gene and loss or mutation results in altered DNA 5hmC and 5mC modifications [11], which may disrupt the germinal center reaction or deregulate B-cell receptor signaling [12]. We also found mutations in other epigenetic modifiers such as $E Z H 2$ and $K M T 2 D$ (mutated in 11 and $26 \%$ of cases, respectively) in tNLPHL.

There are some similarities of tNLPHL to transformed follicular lymphoma (tFL). Both FL and NLPHL have a rich tumor microenvironment (TME) but in transformation to DLBCL, much of the TME is lost and, thus, the dependence on this interaction is altered. Genes more frequently mutated in tFL compared with FL include TP53, EZH2, MYC, CCND3, KMT2C, and $C A R D 11$ [13]. Importantly, TP53 alterations do not appear to be common in tNLPHL, as we saw mutation in only one case and no deletions were detected by CNA. Another notable difference is the low frequency of mutations affecting genes associated with immune surveillance (B2M, MHC class I and II, CD58) in tNLPHL. However, four cases had gains 
in the region of $P D-L 1$ and $P D-L 2$ by CNA which has been seen in NLPHL as well cHL. Unfortunately, we were unable to enrich for LP cells in our cases of NLPHL and, thus, we could not confirm this finding on our cases without transformation.

A limitation to this study is that we did not perform sequencing of the NLPHL cells and, therefore, we cannot determine if these mutations were acquired or were already present at the NLPHL stage of disease. Prior studies have sequenced the LP cells of NLPHL but this was done with a very limited panel $[2,3]$ as compared with our mutation panel that covers the majority of genes implicated in lymphoma. In regard to the unpaired samples, some mutations may represent germline variants (Figs. S2 and S3) since our unpaired cases showed a higher mutation load, but recurrent genes mutated in B-cell lymphoma were selected (Fig. 2).

In summary, this is the largest series of tNLPHL with a conventional DLBCL growth pattern analyzed by CNA and mutational analysis. These lesions are rare but are very informative since they are highly enriched in tumor cells. We found that tNLPHL has a mutational spectrum simulating the DLBCL Cluster 4 of Chapuy et al. [4] with frequent mutations affecting the PI3K pathway (SGK1, ZFP36L1), the NF-kB pathway (CARD11, TNFAIP3, NFKBIA), and linker histones. On the other hand, BRAF/JAK/STAT3 mutations were uncommon in our cases, whereas there were frequent $\mathrm{CN}$ gains affecting $R E L$ and $\mathrm{CN}$ losses involving $C D K N 2 A$. Transformed NLPHLs are also distinctive in enrichment of mutations in genes uncommonly altered in sporadic DLBCL, including TET2, JUNB and NOTCH2. Interestingly, distinct from tFL, TP53 abnormalities and mutations affecting immune surveillance genes are uncommonly observed. This information provides new insights into the biology of tNLPHL and may highlight potential targets for therapy in the future.

\section{Supplementary Material}

Refer to Web version on PubMed Central for supplementary material.

\section{References}

1. Al-Mansour M, Connors JM, Gascoyne RD, Skinnider B, Savage KJ. Transformation to aggressive lymphoma in nodular lymphocyte-predominant Hodgkin's lymphoma. J Clin Oncol. 2010;28:7939. [PubMed: 20048177]

2. Hartmann S, Schuhmacher B, Rausch T, Fuller L, Doring C, Weniger M, et al. Highly recurrent mutations of SGK1, DUSP2 and JUNB in nodular lymphocyte predominant Hodgkin lymphoma. Leukemia. 2016;30:844-53. [PubMed: 26658840]

3. Schuhmacher B, Bein J, Rausch T, Benes V, Tousseyn T, Vornanen M, et al. JUNB, DUSP2, SGK1, SOCS1 and CREBBP are frequently mutated in T-cell/histiocyte-rich large B-cell lymphoma. Haematologica. 2019;104:330-7. [PubMed: 30213827]

4. Chapuy B, Stewart C, Dunford AJ, Kim J, Kamburov A, Redd RA, et al. Molecular subtypes of diffuse large B cell lymphoma are associated with distinct pathogenic mechanisms and outcomes. Nat Med. 2018;24:679-90. [PubMed: 29713087]

5. Schmitz R, Wright GW, Huang DW, Johnson CA, Phelan JD, Wang JQ, et al. Genetics and pathogenesis of diffuse large B-cell lymphoma. N. Engl J Med. 2018;378:1396-407. [PubMed: 29641966] 
6. Reddy A, Zhang J, Davis NS, Moffitt AB, Love CL, Waldrop A, et al. Genetic and functional drivers of diffuse large B cell lymphoma. Cell. 2017;171:481-494 e415. [PubMed: 28985567]

7. Engelsberg A, Kobelt F, Kuhl D. The N-terminus of the serum- and glucocorticoid-inducible kinase Sgk1 specifies mitochondrial localization and rapid turnover. Biochemical J. 2006;399: 69-76.

8. Cordas E, Naray-Fejes-Toth A, Fejes-Toth G. Subcellular location of serum- and glucocorticoidinduced kinase-1 in renal and mammary epithelial cells. Am J Physiol Cell Physiol. 2007;292: C1971-1981. [PubMed: 17202226]

9. Szremska AP, Kenner L, Weisz E, Ott RG, Passegué E, Artwohl M, et al. JunB inhibits proliferation and transformation in B-lymphoid cells. Blood. 2003;102:4159-65. [PubMed: 12907453]

10. Heise N, De Silva NS, Silva K, Carette A, Simonetti G, Pasparakis M, et al. Germinal center B cell maintenance and differentiation are controlled by distinct NF-kappaB transcription factor subunits. J Exp Med. 2014;211:2103-18. [PubMed: 25180063]

11. Dominguez PM, Ghamlouch H, Rosikiewicz W, Kumar P, Béguelin W, Fontán L, et al. TET2 deficiency causes germinal center hyperplasia, impairs plasma cell differentiation, and promotes B-cell lymphomagenesis. Cancer Discov. 2018;8: 1632-53. [PubMed: 30274972]

12. Mouly E, Ghamlouch H, Della-Valle V, Scourzic L, Quivoron C, Roos-Weil D, et al. B-cell tumor development in Tet2-deficient mice. Blood Adv. 2018;2:703-14. [PubMed: 29581109]

13. Bouska A, Zhang W, Gong Q, Iqbal J, Scuto A, Vose J, et al. Combined copy number and mutation analysis identifies oncogenic pathways associated with transformation of follicular lymphoma. Leukemia. 2016;31:83. [PubMed: 27389057] 


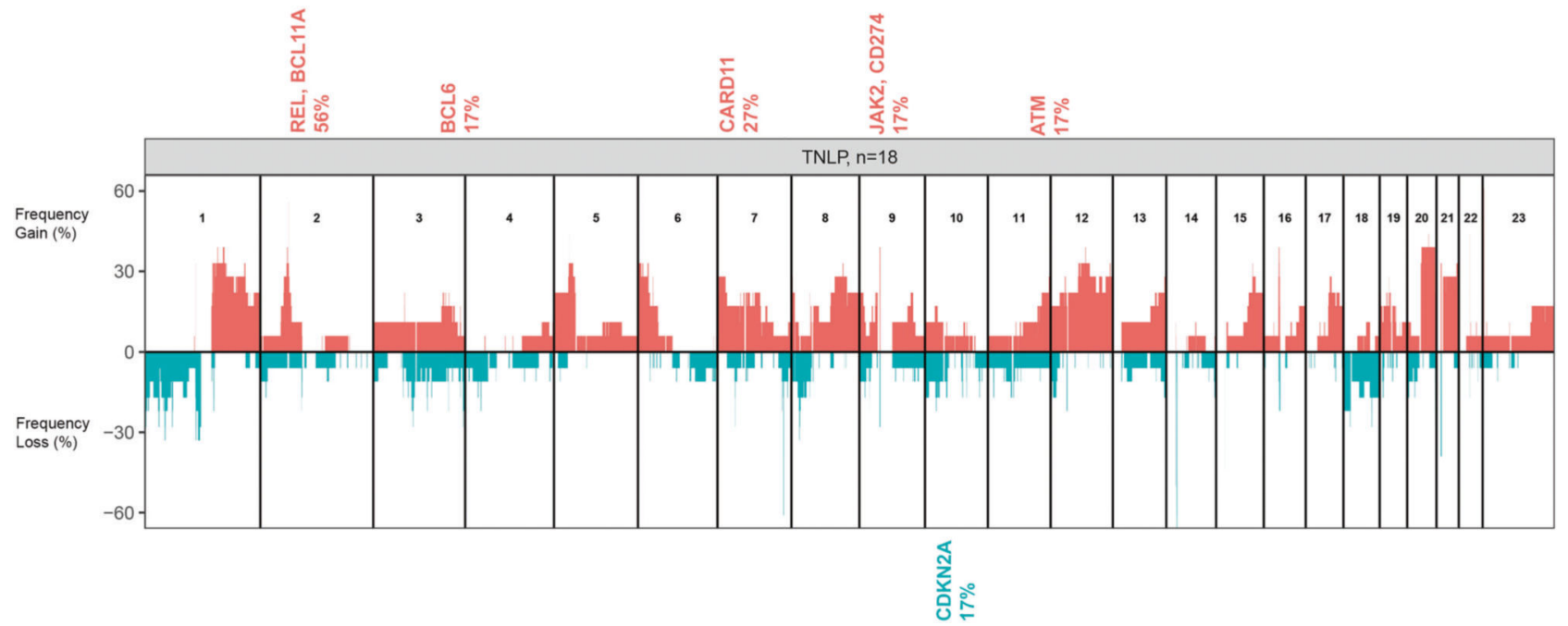

Fig. 1. Copy number analysis of $\mathbf{1 8}$ cases of transformed nodular lymphocyte-predominant Hodgkin lymphoma.

We found copy number gains of several oncogenes (REL, BCL11A, BCL6, CARD11, $J A K 2$ ) and copy number loss of the tumor suppressor gene $C D K N 2 A$ as significant targets within the aberrant locus (Q-bound $<0.05$ and G-score $<1.0$ ) in tNLPHL using GISTIC. 

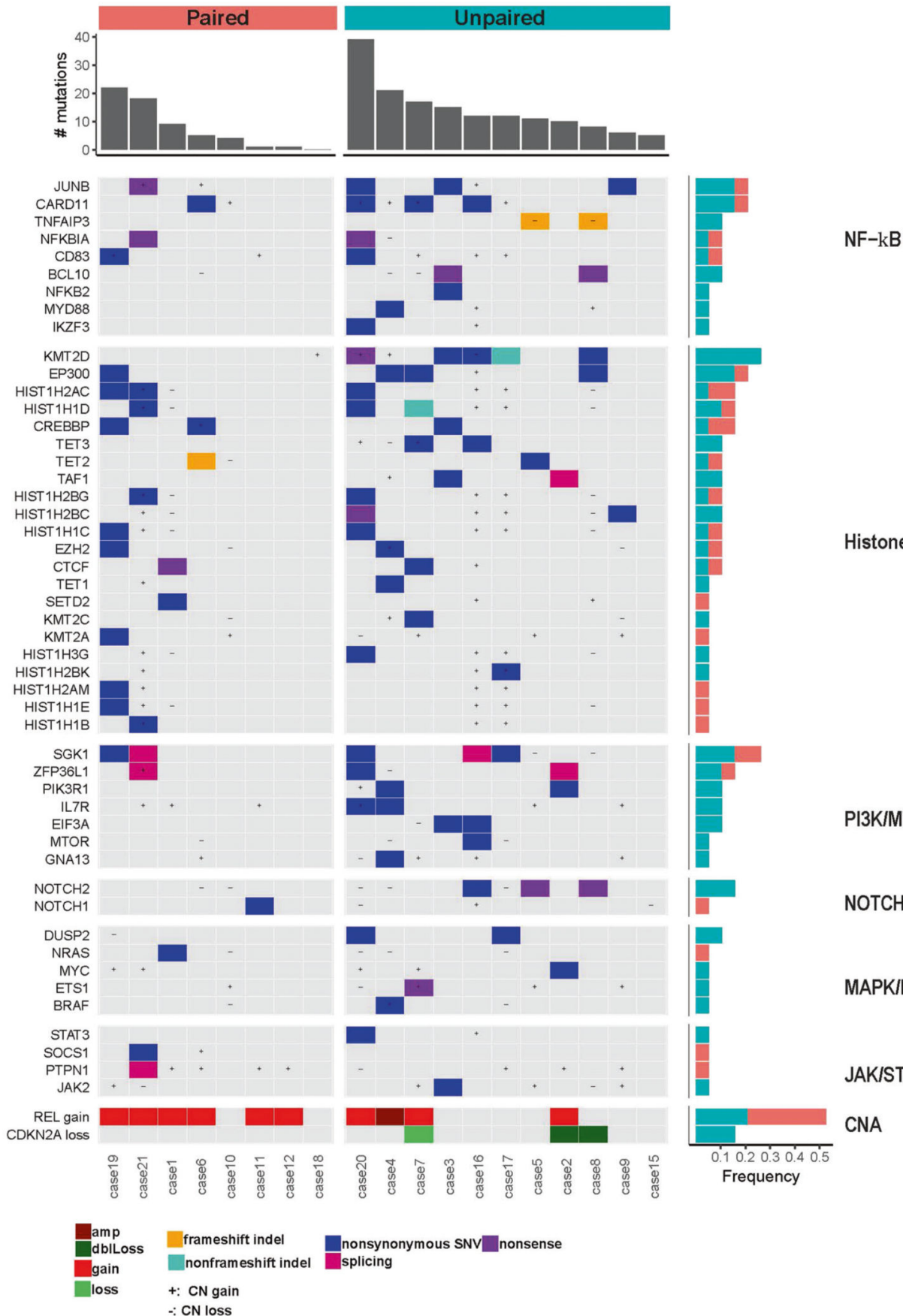

Histone/Epigenetic

PI3KIMTOR

NOTCH

MAPKJMYC

JAKISTAT

CNA

Fig. 2. Select copy number abnormalities and mutation analysis of 19 cases (8 paired and 11 unpaired) of tNLPHL.

Mutations and copy number abnormalities involved key pathways such as NF-kB, PI3K,

NOTCH, MAPK, and JAK/STAT. Histone modifiers were also commonly mutated. The $R E L$ gene was commonly gained as well as loss of $C D K N 2 A$. +, copy number gain; -, copy number loss; amp, amplification; dblLoss, double loss. Paired cases (salmon colored) are ones with corresponding germline DNA used to remove germline variants. Unpaired cases 
(teal colored) are those without corresponding germline DNA. Frequency of these mutations and copy number abnormalities are seen on the left by bar graphs. 